\title{
MULTIPOINT-TO-POINT AND POINT-TO-MULTIPOINT SPACE-TIME NETWORK CODING
}

\author{
Hung-Quoc Lai ${ }^{1,2}$ and K. J. Ray Liü ${ }^{2}$ \\ ${ }^{1}$ US Army RDECOM CERDEC, RDER-STW-N, Aberdeen Proving Ground, MD 21005, USA. \\ ${ }^{2}$ Department of Electrical and Computer Engineering,University of Maryland, College Park, MD 20742, USA.
}

\begin{abstract}
Traditional cooperative communications can improve communication reliability. However, the simultaneous transmissions from multiple relays are challenging in practice due to the issue of imperfect frequency and timing synchronization. In this work, we propose space-time network codes (STNCs) that overcome the issue of imperfect synchronization, reduce the large transmission delay, and still provide full spatial diversity in multipoint-to-point (M2P) and point-to-multipoint $(\mathrm{P} 2 \mathrm{M})$ transmissions. Relays form single linearly-coded signals from the overheard symbols and transmit them to the destination, where multiuser detection is utilized to detect the desired symbol. For a network of $N$ client nodes, M2P and P2M STNCs result in a diversity order of $N$ with only $2 N$ time slots, a significant reduction from $N^{2}$ time slots in traditional cooperative communications using TDMA.
\end{abstract}

Index Terms - Space-time network coding, cooperative communications, frequency synchronization, timing synchronization

\section{INTRODUCTION}

It is well-known that performance of communication systems degrades greatly when operating in radio frequency $(\mathrm{RF})$ environments characterized by multipath propagation such as urban environments. Diversity techniques such as time diversity, frequency diversity, and spatial diversity can improve transmission reliability. Among these techniques, spatial diversity achieved by cooperative communications [1] has become recently attractive.

In cooperative communications, nodes acting as relays retransmit the overheard information to a destination. The distributed antennas among nodes are used to provide spatial diversity without the need to use multiple antennas at the source. Various cooperative diversity protocols have been proposed and analyzed in [2]-[9]. They often consist of two phases: source transmission and relay transmission. In the first phase, a source broadcasts its information to a destination and the relays, which then forward the overheard information to the destination in the second phase. The simultaneous transmissions from two or more relays are challenging in practice due to the imperfect frequency and timing synchronization, especially in mobile conditions where relays move at different speeds and in different directions.

In this work, we propose space-time network codes (STNCs) that overcome the issue of imperfect synchronization, reduce the large transmission delay, and still provide full spatial diversity for multipoint-to-point (M2P) and point-tomultipoint (P2M) transmissions. Relay nodes in a network form single linearly-coded signals from the overheard symbols and transmit them to the destination, where multiuser detection is utilized to detect the desired symbol. For a network of $N$ cooperating nodes, $\mathrm{M} 2 \mathrm{P}$ and $\mathrm{P} 2 \mathrm{M}$ STNCs require only $2 N$ time slots, a substantial reduction from $N^{2}$ time slots in traditional cooperative communications using TDMA [7], to achieve a spatial diversity order of $N$ for each transmitted symbol. Both decode-and-forward (DF) and amplify-andforward (AF) protocols in cooperative communications are considered. We derive the exact and the asymptotic symbolerror-rate (SER) expressions for general M-PSK modulation for DF protocol. For AF protocol, we offer the conditional SER expression given the channel knowledge. Simulations are provided to confirm the performance analysis.

The rest of this paper is organized as follows. After this introduction section, M2P and P2M STNCs are introduced in Section 2. Signal detection is followed in Section 3. The performance analysis is presented in Section 4. The simulations to confirm the analysis are also provided in Section 4. Lastly, we draw some conclusions in Section 5.

\section{M2P AND P2M STNCS}

We consider a wireless network consisting of $(N+1)$ nodes denoted as $U_{0}, U_{1}, U_{2}, \ldots, U_{N}$, where $U_{0}$ is a base node and $U_{1}, U_{2}, \ldots, U_{N}$ are client nodes in $\mathrm{M} 2 \mathrm{P}$ and $\mathrm{P} 2 \mathrm{M}$ setting. The channels between any two nodes, denoted as $h_{u v}$, are modeled as narrow-band Rayleigh fading with additive white Gaussian noise $(\mathrm{AWGN})$, i.e., that $h_{u v} \sim \mathcal{C N}\left(0, \sigma_{u v}^{2}\right)$ with the channel variance $\sigma_{u v}^{2}$. Quasi-static channels are assumed, where the channels remain constant over each time slot and change independently between consecutive slots.

To protect transmitted symbols from interference, we use complex-valued signature waveforms. The cross-correlation between two signature waveforms $s_{j}(t)$ and $s_{i}(t)$ is $\rho_{j i}=$ $\left\langle s_{j}(t), s_{i}(t)\right\rangle$, where $\langle f(t), g(t)\rangle \triangleq \int_{0}^{T} f(t) g^{*}(t) d t$ is the inner product between $f(t)$ and $g(t)$ with the symbol interval $T$. Each node is assumed to know all the signature waveforms of other nodes.

In M2P transmission, $U_{1}, U_{2}, \ldots, U_{N}$ wish to transmit symbols $x_{1}, x_{2}, \ldots, x_{N}$, respectively, to $U_{0}$. The STNC comprises source and relay transmission phases and can be represented by matrices

\begin{tabular}{|c|c|c|c|c|c|}
\hline & $U_{1}$ & $\cdots$ & $U_{j}$ & $\ldots$ & $U_{N}$ \\
\hline$T_{1}$ & $a_{11} x_{1}$ & $\ldots$ & 0 & $\ldots$ & 0 \\
\hline$\vdots$ &. & 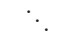 & $\vdots$ & $\ldots$ & $\vdots$ \\
\hline$T_{j}$ & 0 & $\ldots$ & $a_{j j} x_{j}$ & $\cdots$ & 0 \\
\hline & : & $\ldots$ & $\vdots$ & $\ddots$ & $\vdots$ \\
\hline$N$ & 0 & $\ldots$ & 0 & $\ldots$ & $a_{N N} x_{N}$ \\
\hline
\end{tabular}

and

\begin{tabular}{|c|c|c|c|c|}
\hline & $U_{1}$ & $U_{i}$ & $\ldots$ & $U_{N}$ \\
\hline$T_{1}$ & $\sum_{k \neq 1} a_{1 k} x_{k}$ & $\cdots$ & $\cdots$ & 0 \\
\hline . & $\vdots$ & $\ddots$ & $\ldots$ & : \\
\hline$T_{i}$ & 0 & $\cdots \sum_{k \neq i} a_{i k} x_{k}$ & $\cdots$ & 0 \\
\hline. & $\vdots$ & $\ldots$ & & $\vdots$ \\
\hline$T_{N}$ & 0 & $\ldots$ & $\cdots \sum$ & ${ }_{N} a_{N k}$ \\
\hline
\end{tabular}

respectively, where $a_{i j}$ 's are signal coefficients and will be clarified later and $T_{i}$ denotes the $i$ th time slot. From the STNC, $2 N$ time slots are required for transmissions of the $N$ symbols to $U_{0}$. In the source transmission phase, each client node $U_{j}$ for $j=1,2, \ldots, N$ is assigned a time slot, denoted 
$T_{j}$, to broadcast its symbol $x_{j}$ to the base node $U_{0}$ and other client nodes $U_{i}, i \neq j$. After this phase, $U_{i}$ possesses a set of $(N-1)$ symbols $x_{1}, \ldots, x_{k}, \ldots, x_{N}, k \neq i$, from which it forms a single linearly-coded signal. $U_{i}$ can detect the symbols and re-encode them if the decoding is successful [7], the so called DF protocol, or it simply amplifies the signals and combines them to form the single signal, the so called AF protocol. Note that this DF scheme follows selective-relaying protocol in the literature [4]. In the relay transmission phase, $U_{i}$ transmits the coded signal to $U_{0}$ in its own dedicated time slot, denoted $T_{i}$.

In contrast with M2P, the single base node $U_{0}$ in $\mathrm{P} 2 \mathrm{M}$ wants to transmit symbols $x_{1}, x_{2}, \ldots, x_{N}$ to $U_{1}, U_{2}, \ldots, U_{N}$, respectively. The STNC also consists of source and relay transmission phases and can be represented by matrices

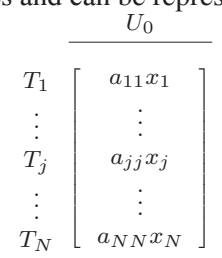

and

\begin{tabular}{|c|c|c|c|c|}
\hline & $U_{1}$ & $U_{i}$ & $\ldots$ & $U_{N}$ \\
\hline$T_{1}$ & $\sum_{k \neq 1} a_{1 k} x_{k}$ & 0 & .. & 0 \\
\hline$T_{i}$ & $\vdots$ & $\sum_{k \neq i} a_{i k} x_{k}$ & & $\vdots$ \\
\hline & $\vdots$ & $\vdots$ & & $\vdots$ \\
\hline$T_{N}$ & 0 & 0 & $\cdots \sum$ & ${ }_{N} a_{N k} x_{k}$ \\
\hline
\end{tabular}

respectively, where the client nodes help one another in relaying transmitted symbols. As shown in (1)-(4), M2P and P2M STNCs have a similar form; the difference is in the origin of the source signal and the destination of the relaying signals. Due to the space limitation, we only present signal models, signal detection and performance analysis for M2P STNC.

An arbitrary symbol $x_{j}, j=1,2, \ldots, N$, in $\mathrm{M} 2 \mathrm{P}$ is transmitted $N$ times as shown in (1) and (2) by the source node and the relay nodes, and its associated transmit power $P_{j}=$ $\sum_{i=1}^{N} P_{i j}$ is distributed among the $N$ transmissions, where $P_{j j}$ and $P_{i j}$ are the power allocated at the source node $U_{j}$ and a relay node $U_{i}$, respectively. The received signals at $U_{0}$ and $U_{i}$ in the source transmission phase are

$$
y_{j 0}(t)=h_{j 0} \sqrt{P_{j j}} x_{j} s_{j}(t)+w_{j 0}(t)
$$

and

$$
y_{j i}(t)=h_{j i} \sqrt{P_{j j}} x_{j} s_{j}(t)+w_{j i}(t),
$$

respectively, where $w_{j 0}(t)$ and $w_{j i}(t)$ are zero-mean and $N_{0^{-}}$ variance AWGN. The received signal at $U_{0}$ from $U_{i}$ in the relay transmission phase is

$$
y_{i 0}(t)=h_{i 0} \sum_{\substack{k=1 \\ k \neq i}}^{N} \sqrt{\widetilde{P}_{i k}} x_{k} s_{k}(t)+w_{i 0}(t)
$$

including symbol $x_{j}$ when $k=j$. In (7),

$$
\widetilde{P}_{i k}= \begin{cases}P_{i k} & \text { if } U_{i} \text { decodes } x_{k} \text { correctly } \\ 0 & \text { otherwise }\end{cases}
$$

for the case of DF and

$$
\widetilde{P}_{i k}=\frac{P_{i k} P_{k k}\left|h_{k i}\right|^{2}}{P_{k k}\left|h_{k i}\right|^{2}+N_{0}}
$$

for the case of $\mathrm{AF}$ and $w_{i j}(t)$ is zero-mean and $N_{0} f_{i 0^{-}}$ variance AWGN, where

$$
f_{i 0}= \begin{cases}1 & \text { for DF } \\ 1+\sum_{\substack{k=1 \\ k \neq i}}^{N} \frac{P_{i k}\left|h_{i 0}\right|^{2}}{P_{k k}\left|h_{k i}\right|^{2}+N_{0}} & \text { for AF }\end{cases}
$$

is a factor representing the impact on $U_{0}$ due to the noise amplification at $U_{i}$.

\section{SIGNAL DETECTION}

To detect a desired symbol, a destination is assumed to have a full knowledge of the channel state information (CSI), which can be acquired using a preamble in the transmitted signal as usually done in practice such as IEEE 802.11 systems [10]. In the case of DF, a receiver is also assumed to know the decoding correctness (or the detection state) at the relay nodes. This can be done by using an $N$-bit indicator in the relaying signal, each bit dedicated to each transmitted symbol.

In M2P, $U_{0}$ detects an arbitrary symbol $x_{j}$ based on $N$ soft symbols extracted from $N$ received signals containing the symbol. The first soft symbol of $x_{j}$ comes directly from the source node $U_{j}$ by applying matched-filtering to signal $y_{j 0}(t)$ in (5) with respect to signature waveform $s_{j}(t)$ to obtain

$$
y_{j 0 j}=\left\langle y_{j 0}(t), s_{j}(t)\right\rangle=h_{j 0} \sqrt{P_{j j}} x_{j}+w_{j 0 j} .
$$

The remaining $(N-1)$ soft symbols are collected from $(N-$ 1 ) relaying signals $y_{i 0}(t)$ for $i \neq j$ in (7) as follows. For each signal $y_{i 0}(t), U_{0}$ applies a bank of matched-filtering to the signal with respect to signature waveforms $s_{m}(t), m=$ $1,2, \ldots, N$ and $m \neq i$, to obtain

$$
y_{i 0 m}=\left\langle y_{i 0}(t), s_{m}(t)\right\rangle=h_{i 0} \sum_{\substack{k=1 \\ k \neq i}}^{N} \sqrt{\widetilde{P}_{i k}} x_{k} \rho_{k m}+w_{i 0 m} .
$$

Then it forms an $(N-1) \times 1$ vector comprising the $y_{i 0 m}$ 's as

$$
\mathbf{y}_{i 0}=h_{i 0} \mathbf{R}_{i} \mathbf{A}_{i} \mathbf{x}_{i}+\mathbf{w}_{i 0},
$$

where $\mathbf{y}_{i 0}=\left[y_{i 01}, \ldots, y_{i 0(i-1)}, y_{i 0(i+1)}, \ldots, y_{i 0 N}\right]^{T}, \mathbf{x}_{i}=$ $\left[x_{1}, \ldots, x_{i-1}, x_{i+1} \ldots, x_{N}\right]^{T}, \mathbf{w}_{i 0}=\left[w_{i 01}, \ldots, w_{i 0(i-1)}\right.$, $\left.w_{i 0(i+1)}, \ldots, w_{i 0 N}\right]^{T} \sim \mathcal{C N}\left(\mathbf{0}, N_{0} f_{i 0} \mathbf{R}_{i}\right)$ with $f_{i 0}$ in (10),

$\mathbf{R}_{i}=\left[\begin{array}{cccccc}1 & \cdots & \rho_{(i-1) 1} & \rho_{(i+1) 1} & \cdots & \rho_{N 1} \\ \vdots & \ddots & \vdots & \vdots & \cdots & \vdots \\ \rho_{1(i-1)} & \cdots & 1 & \rho_{(i+1)(i-1)} & \cdots & \rho_{N(i-1)} \\ \rho_{1(i+1)} & \cdots & \rho_{(i-1)(i+1)} & 1 & \cdots & \rho_{N(i+1)} \\ \vdots & \cdots & \vdots & \vdots & \ddots & \vdots \\ \rho_{1 N} & \cdots & \rho_{(i-1) N} & \rho_{(i+1) N} & \cdots & 1\end{array}\right]$,

and $\mathbf{A}_{i}=\operatorname{diag}\left\{\sqrt{\widetilde{P}_{i 1}}, \ldots, \sqrt{\widetilde{P}_{i(i-1)}}, \sqrt{\widetilde{P}_{i(i+1)}}, \ldots \sqrt{\widetilde{P}_{i N}}\right\}$ with $\operatorname{diag}\{$.$\} denoting an diagonal matrix. Note that we have ex-$ plicitly expressed the above equalities in terms of the index $i$, not in terms of the indices $k$ and $m$ as in (12). Note further that the soft symbol of $x_{j}$ from this client node corresponds to the $j$ th row for $i>j$ or the $(j-1)$ st row for $i<j$ in the above equalities. The signal vector $\mathbf{y}_{i 0}$ is then decorrelated, assuming that $\mathbf{R}_{i}$ is invertible with the inverse matrix $\mathbf{R}_{i}^{-1}$ to obtain

$$
\widetilde{\mathbf{y}}_{i 0}=\mathbf{R}_{i}^{-1} \mathbf{y}_{i 0}=h_{i 0} \mathbf{A}_{i} \mathbf{x}_{i}+\widetilde{\mathbf{w}}_{i 0},
$$

where $\widetilde{\mathbf{w}}_{i 0} \sim \mathcal{C N}\left(\mathbf{0}, N_{0} f_{i 0} \mathbf{R}_{i}^{-1}\right)$. Since $\mathbf{A}_{i}$ is a diagonal matrix, the soft symbol of $x_{j}$ from $U_{i}$ is

$$
\widetilde{y}_{i 0 j}=h_{i 0} \sqrt{\widetilde{P}_{i j}} x_{j}+\widetilde{w}_{i 0 j},
$$

where $\widetilde{w}_{i 0} \sim \mathcal{C N}\left(0, N_{0} f_{i 0} r_{i j}\right)$ with $r_{i j}$, the diagonal element of matrix $\mathbf{R}_{i}^{-1}$ associated with symbol $x_{j}$. That is the $j$ th or the $(j-1)$ st diagonal element of the inverse matrix for $i>j$ or $i<j$, respectively. Since there are $(N-1)$ relaying signals from $U_{i}, i=1,2, \ldots, N$ and $i \neq j, U_{0}$ obtains $(N-1)$ soft symbols of $x_{j}$ in the above manner.

Next, from the $N$ soft symbols of $x_{j}$ in (11) and (16), $U_{0}$ forms an $N \times 1$ signal vector

$$
\mathbf{y}_{j 0}=\mathbf{a}_{j 0} x_{j}+\mathbf{w}_{j 0},
$$

where $\mathbf{a}_{j 0}=\left[h_{10} \sqrt{\widetilde{P}_{1 j}}, \ldots, h_{j 0} \sqrt{P_{j j}}, \ldots, h_{N 0} \sqrt{\widetilde{P}_{N j}}\right]^{T}$ and $\mathbf{w}_{j 0} \sim \mathcal{C N}\left(0, \mathbf{K}_{j 0}\right)$. We can show that $\mathbf{K}_{j 0}=\operatorname{diag}\left\{N_{0} f_{10} r_{1 j}\right.$, 
$\left.\ldots, N_{0}, \ldots, N_{0} f_{N 0} r_{N j}\right\}$. Let $\mathbf{b}_{j 0}=\left[\frac{h_{10} \sqrt{\widetilde{P}_{1 j}}}{N_{0} f_{10} r_{1 j}}, \ldots, \frac{h_{j 0} \sqrt{P_{j j}}}{N_{0}}, \ldots\right.$, $\left.\frac{h_{N 0} \sqrt{\widetilde{P}_{N j}}}{N_{0} f_{N 0} r_{N j}}\right]^{T}$. Then the desired symbol $x_{j}$ can be detected based on

$$
\widehat{x}_{j 0} \triangleq \mathbf{b}_{j 0}^{H} \mathbf{y}_{j 0}=a_{j 0} x_{j}+w_{j 0}
$$

where

$$
a_{j 0} \triangleq \mathbf{b}_{j 0}^{H} \mathbf{a}_{j 0}=\frac{P_{j j}\left|h_{j 0}\right|^{2}}{N_{0}}+\sum_{\substack{i=1 \\ i \neq j}}^{N} \frac{\widetilde{P}_{i j}\left|h_{i 0}\right|^{2}}{N_{0} f_{i 0} r_{i j}}
$$

and $w_{j 0} \triangleq \mathbf{b}_{j 0}^{H} \mathbf{w}_{j 0} \sim \mathcal{C N}\left(0, \sigma_{j 0}^{2}\right)$ with $\sigma_{j 0}^{2}=a_{j 0}$.

The detection of $x_{j}$ at the relay node $U_{i}$ can follow a matched-filtering to signal $y_{j i}(t)$ in (6) with respect to the signature waveform $s_{j}(t)$ as

$$
y_{j i}=\left\langle y_{j i}(t), s_{j}(t)\right\rangle=h_{j i} \sqrt{P_{j j}} x_{j}+w_{j i}
$$

where $w_{j i} \sim \mathcal{C N}\left(0, N_{0}\right)$.

\section{PERFORMANCE ANALYSIS AND SIMULATIONS}

\subsection{An Exact SER Expression}

For M2P STNC, the detection in (18) provides the maximal conditional signal-to-interference-plus-noise ratio (SINR) $\gamma_{j 0}$ corresponding to the desired symbol $x_{j}$ as

$$
\gamma_{j 0}=\frac{a_{j 0}^{2}}{\sigma_{j 0}^{2}}=\frac{P_{j j}\left|h_{j 0}\right|^{2}}{N_{0}}+\sum_{\substack{i=1 \\ i \neq j}}^{N} \frac{\widetilde{P}_{i j}\left|h_{i 0}\right|^{2}}{N_{0} f_{i 0} r_{i j}} .
$$

For DF protocol, let $\beta_{i j} \in\{0,1\}, i=1,2, \ldots, N$ and $i \neq j$, represent a detection state at $U_{i}$, a success or a failure in detecting of $x_{j}$. Because $U_{i}$ forwards $x_{j}$ only if it has successfully detected the symbol, $\widetilde{P}_{i j}=P_{i j} \beta_{i j}$. All $\beta_{i j}$ 's form a decimal number $S_{j}=\left[\beta_{1 j} \ldots \beta_{i j} \ldots \beta_{N j}\right]_{2}$, where $[.]_{2}$ denotes a base- 2 number, that represents one of $2^{(N-1)}$ detection states of the $(N-1)$ client nodes $U_{i}$ 's acting as relays. Because the detection is independent from one relay node to the others, $\beta_{i j}$ 's are independent Bernoulli random variables with a distribution

$$
G\left(\beta_{i j}\right)=\left\{\begin{array}{ll}
1-S E R_{j i} & \text { if } \beta_{i j}=1 \\
S E R_{j i} & \text { if } \beta_{i j}=0
\end{array},\right.
$$

where $S E R_{j i}$ is the SER in detection of $x_{j}$ at $U_{i}$. Hence the probability of $x_{j}$ detection in state $S_{j}$ is

$$
\operatorname{Pr}\left(S_{j}\right)=\prod_{\substack{i=1 \\ i \neq j}}^{N} G\left(\beta_{i j}\right) .
$$

Given a detection state $S_{j}$, we rewrite (21) as

$$
\gamma_{j 0 \mid S_{j}}=\frac{P_{j j}\left|h_{j 0}\right|^{2}}{N_{0}}+\sum_{\substack{i=1 \\ i \neq j}}^{N} \frac{P_{i j} \beta_{i j}\left|h_{i 0}\right|^{2}}{N_{0} r_{i j}},
$$

where we have used $f_{i 0}=1$ for DF protocol.

In general, the conditional SER for M-PSK modulation with conditional signal-to-noise ratio (SNR) $\gamma$ for a generic set of channel coefficients $\left\{h_{u v}\right\}$ is given by [11]

$$
S E R_{\mid\left\{h_{u v}\right\}}=\Psi(\gamma) \triangleq \frac{1}{\pi} \int_{0}^{(M-1) \pi / M} \exp \left(-\frac{b \gamma}{\sin ^{2} \theta}\right) d \theta
$$

where $b=\sin ^{2}(\pi / M)$. Based on (20), the SNR in detection of $x_{j}$ at $U_{i}$ given the channel gain is $\gamma_{j i}=P_{j j}\left|h_{0 i}\right|^{2} / N_{0}$. By averaging (25) with respect to the exponential random variable $\left|h_{j i}\right|^{2}$, the SER in detecting $x_{j}$ at $U_{i}$ can be shown as [11]

$$
S E R_{j i}=F\left(1+\left(b P_{j j} \sigma_{j i}^{2}\right) /\left(N_{0} \sin ^{2} \theta\right)\right),
$$

where

$$
F(x(\theta))=(1 / \pi) \int_{0}^{(M-1) \pi / M}(1 / x(\theta)) d \theta .
$$

Given a detection state $S_{j}$, which can take $2^{(N-1)}$ values, the conditional SER in detecting $x_{j}$ at $U_{j}$ can be calculated using the law of total probability [12] as

$$
S E R_{j \mid\left\{h_{j 0}, h_{i 0}\right\}}=\sum_{S_{j}=0}^{2^{(N-1)}-1} \operatorname{Pr}\left(\hat{x}_{j} \neq x_{j} \mid S_{j}\right) \cdot \operatorname{Pr}\left(S_{j}\right),
$$

where $\operatorname{Pr}\left(S_{j}\right)$ follows (23) and

$$
\operatorname{Pr}\left(\hat{x}_{j} \neq x_{j} \mid S_{j}\right)=\Psi\left(\gamma_{j 0 \mid S_{j}}\right)
$$

with $\gamma_{j 0 \mid S_{j}}$ following (24). By averaging (28) with respect to the exponential random variables $\left\{\left|h_{j 0}\right|^{2},\left|h_{i 0}\right|^{2}\right\}$, the exact SER in detecting $x_{j}$ at $U_{0}$ can be given by [11]

$$
\begin{aligned}
S E R_{j}= & \sum_{S_{j}=0}^{2^{(N-1)}-1} F\left(\left(1+\frac{b P_{j j} \sigma_{j 0}^{2}}{N_{0} \sin ^{2} \theta}\right) \times\right. \\
& \left.\prod_{\substack{i=1 \\
i \neq j}}^{N}\left(1+\frac{b P_{i j} \beta_{i j} \sigma_{i 0}^{2}}{N_{0} r_{i j} \sin ^{2} \theta}\right)\right) \prod_{\substack{i=1 \\
i \neq j}}^{N} G\left(\beta_{i j}\right),
\end{aligned}
$$

where $G($.$) and F($.$) follows (22) and (27), respectively.$

For AF protocol, the conditional SER is

$$
S E R_{j \mid\left\{h_{0 i j}, h_{i j}\right\}}=\Psi\left(\gamma_{j}\right) \text {, }
$$

where $\Psi($.$) is defined in (25) and \gamma_{j}$ follows (21) with $f_{i j}$ in (10) for AF protocol.

\subsection{An Asymptotic SER Expression}

To obtain the asymptotic SER performance, i.e., performance at high SNR, two approximations are required. First, $S E R_{j i}$ is sufficiently small compared to 1 , and thus assumably ( 1 $\left.S E R_{j i}\right) \simeq 1$. Second, the 1's in the argument of $F($.$) can be$ ignored. Hence we rewrite (30) as

$$
\begin{aligned}
S E R_{j} \simeq \sum_{S_{j}=0}^{2^{(N-1)}-1} F(\left(\frac{b P_{j} \alpha_{j j} \sigma_{j 0}^{2}}{N_{0} \sin ^{2} \theta}\right) \underbrace{N}_{\substack{i=1 ; i \neq j \\
\beta_{i j}=1}}\left(\frac{b P_{j} \alpha_{i j} \sigma_{i 0}^{2}}{N_{0} r_{i j} \sin ^{2} \theta}\right)) & \underbrace{}_{\mathcal{A}} \\
& \times \underbrace{\prod_{\mathcal{B}}^{N} F\left(\frac{b P_{j} \alpha_{j j} \sigma_{j i}^{2}}{N_{0} \sin ^{2} \theta}\right)}_{\substack{i=1 ; i \neq j \\
\beta_{i j}=0}},
\end{aligned}
$$

where $\alpha_{j j} \triangleq \frac{P_{j j}}{P_{j}}$ and $\alpha_{i j} \triangleq \frac{P_{i j}}{P_{j}}, \sum_{i=1}^{N} \alpha_{i j}=1$, denote the fraction of power $P_{j}$ allocated at the source node $U_{j}$ and a relay node $U_{i}$.

Let $\Omega_{j 0}$ and $\Omega_{j 1}$ denote subsets of the indices of nodes that decode $x_{j}$ erroneously and correctly, respectively. Then $\Omega_{j 0}=\left\{i: \beta_{i j}=0\right\}$ and $\Omega_{j 1}=\left\{i: \beta_{i j}=1\right\}$. Furthermore, $\left|\Omega_{j 0}\right|$ and $\left|\Omega_{j 1}\right| \in\{0,1, \ldots,(N-1)\}$, and $\left|\Omega_{j 0}\right|+\left|\Omega_{j 1}\right|=$ $(N-1)$ for any detection state $S_{j}$. Hence in (32), we can show that

$$
\begin{gathered}
\mathcal{A} \simeq\left(\frac{N_{0}}{b P_{j}}\right)^{1+\left|\Omega_{j 1}\right|} \frac{g\left(1+\left|\Omega_{j 1}\right|\right)}{\alpha_{j j} \sigma_{j 0}^{2} \prod_{i \in \Omega_{j 1}} \alpha_{i j}\left(\frac{\sigma_{i 0}^{2}}{r_{i j}}\right)}, \\
\mathcal{B} \simeq\left(\frac{N_{0}}{b P_{j}}\right)^{\left|\Omega_{j 0}\right|} \frac{[g(1)]^{\left|\Omega_{j 0}\right|}}{\alpha_{j j}^{\left|\Omega \Omega_{j 0}\right|} \prod_{i \in \Omega_{j 0}} \sigma_{j i}^{2}},
\end{gathered}
$$

where

$$
g(x)=\frac{1}{\pi} \int_{0}^{(M-1) \pi / M}[\sin (\theta)]^{2 x} d \theta
$$

Consequently, (32) can be rewritten as

$$
\begin{array}{r}
S E R_{j} \simeq\left(\frac{b P_{j}}{N_{0}}\right)^{-N} \frac{1}{\sigma_{j 0}^{2}} \\
\times \sum_{S_{j}=0}^{2^{(N-1)}-1} \frac{g\left(1+\left|\Omega_{j 1}\right|\right)[g(1)]^{\left|\Omega_{j 0}\right|}}{\alpha_{j j}^{1+\left|\Omega_{j 0}\right|} \prod_{i \in \Omega_{j 1}} \alpha_{i j}\left(\frac{\sigma_{i 0}^{2}}{r_{i j}}\right) \prod_{i \in \Omega_{j 0}} \sigma_{j i}^{2}} .
\end{array}
$$




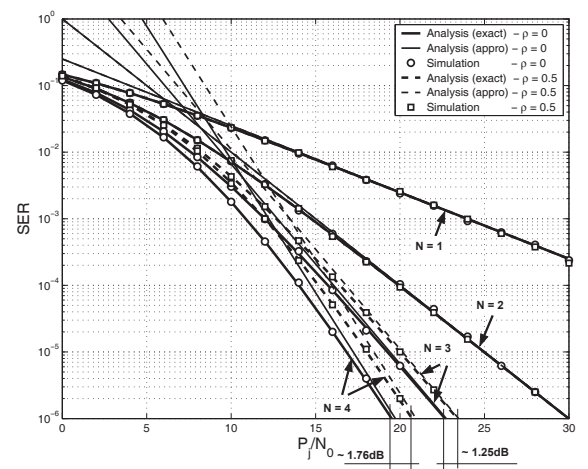

Fig. 1. SER versus SNR performance for BPSK modulation in DF M2P and P2M STNCs.

\subsection{Diversity Order and Interference Impact on SNR} Diversity order of a communication scheme is defined as

$$
\operatorname{div}=-\lim _{\gamma \rightarrow \infty} \frac{\log S E R(\gamma)}{\log \gamma},
$$

where $\operatorname{SER}(\gamma)$ is the SER with the SNR $\gamma \triangleq P / N_{0}$. From (36), $x_{j}$ is clearly received with a full diversity order of $N$.

To see the interference impact on the SNR when using $\rho_{i j} \neq 0$, we consider unique cross-correlations $\rho_{i j}=\rho$ for all $i \neq j$. We can show that

$$
r_{i j}=\frac{1+(N-3) \rho}{(1-\rho)(1+(N-2) \rho)} \triangleq r
$$

the same for all $i$ and $j$. Because $r \geq 1$ and $\left|\Omega_{j 1}\right| \leq N$, $r^{\left|\Omega_{j 1}\right|} \leq r^{N}$. Hence we rewrite (36) as

$$
\begin{array}{r}
S E R_{j} \lesssim\left(\frac{b P_{j}}{N_{0}}\right)^{-N} \frac{r^{N}}{\sigma_{j 0}^{2}} \\
\times \sum_{S_{j}=0}^{{ }^{(N-1)}-1} \frac{g\left(1+\left|\Omega_{j 1}\right|\right)[g(1)]^{\left|\Omega_{j 0}\right|}}{\alpha_{j j}^{1+\left|\Omega_{j 0}\right|} \prod_{i \in \Omega_{j 1}} \alpha_{i 0} \sigma_{i 0}^{2} \prod_{i \in \Omega_{j 0}} \sigma_{j i}^{2}} .
\end{array}
$$

Based on (39), given the same required SER, the loss in SNR when using unique nonorthogonal codes can be shown at most $\Delta \gamma=10 \log r(\mathrm{~dB})$. Furthermore, the maximal loss is

$$
\Delta \gamma_{\max } \triangleq \lim _{N \rightarrow \infty} \Delta \gamma=-10 \log (1-\rho)(\mathrm{dB}) .
$$

\subsection{Simulations}

Figures 1 and 2 present the SER performance for DF and $\mathrm{AF}$ protocols with BPSK modulation. We assume unit variance for AWGN and channel coefficients, i.e., $N_{0}=1$ and $\sigma_{j 0}^{2}=\sigma_{j i}^{2}=1$ for all $j$ and $i \neq j$. Various values of $N=1,2$, 3 , and 4 are used, and the transmit power $P_{j}=\sum_{i=1}^{N} P_{i j}$ corresponding to $x_{j}$ is the same for all $j=1,2, \ldots, N$. Furthermore, we assume equal power allocation [7] with $\alpha_{j j}=1 / 2$ and $\alpha_{i j}=1 /(2(N-1))$ for $i \neq j$. We also assume a unique cross-correlation $\rho_{j i}=\rho$ for all $i \neq j$, and we take $\rho=0$ and $\rho=0.5$ in our simulations.

From the figures, the simulation curves match analytical curves well and that validates our analysis. Moreover, the STNCs clearly provide the expected diversity orders in both $\mathrm{DF}$ and $\mathrm{AF}$ protocols, i.e., that symbol $x_{j}$ is received with diversity order $N$ for $N$ client nodes. The figures also show the SNR gaps between $\rho=0$ and $\rho=0.5$. In particular for the case of $N=4$, it requires about $1.76 \mathrm{~dB}$ and $2 \mathrm{~dB}$ when using $\rho=0.5$ in $\mathrm{DF}$ and $\mathrm{AF}$ protocols, respectively.

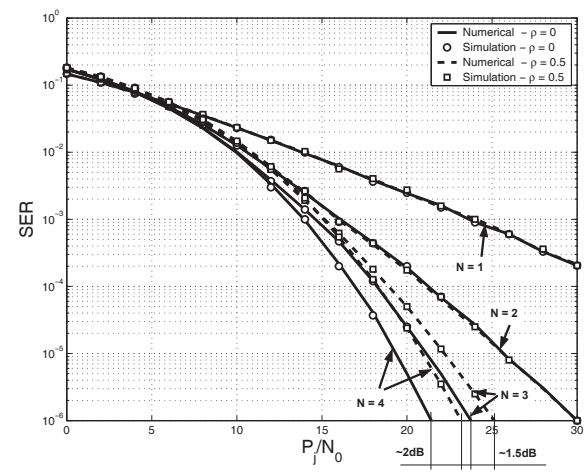

Fig. 2. SER versus SNR performance for BPSK modulation in AF M2P and P2M STNCs.

\section{CONCLUSIONS}

In this paper, we proposed M2P and P2M STNCs to govern the cooperation among nodes in $\mathrm{M} 2 \mathrm{P}$ and $\mathrm{P} 2 \mathrm{M}$ transmissions, respectively. The STNCs utilize cooperative communications and linear network coding to overcome the issue of imperfect frequency and timing synchronization and reduce the large required time slots while still providing full spatial diversity as in traditional cooperative communications. For a network of $N$ client nodes and a base node, M2P and P2M STNCs result in a diversity order of $N$ for each transmitted symbol with a total of $2 N$ time slots, a substantial reduction in comparison with $N^{2}$ time slot required in the traditional cooperative communications using TDMA.

\section{REFERENCES}

[1] K. J. R. Liu, A. K. Sadek, W. Su, and A. Kwasinski, Cooperative Communications and Networking. Cambridge University Press, 2008.

[2] J. N. Laneman and G. W. Wornell, "Distributed space-time coded protocols for exploiting cooperative diversity in wireless networks," IEEE Trans. Inform. Theory, vol. 49, pp. 2415-2525, Oct. 2003.

[3] A. Sendonaris, E. Erkip, and B. Aazhang, "User cooperation diversity - Part I: System description,” IEEE Trans. Comm., vol. 51, pp. 19271938, Nov. 2003.

[4] J. N. Laneman, D. N. C. Tse, and G. W. Wornell, "Cooperative diversity in wireless networks: efficient protocols and outage behavior," IEEE Trans. Inform. Theory, vol. 50, no. 12, pp. 3062-3080, Dec. 2004.

[5] Y. Cao and B. Vojcic, "MMSE multiuser detection for cooperative diversity CDMA systems," Proc. IEEE WCNC, vol. 1, pp. 42-47, Mar. 2004.

[6] L. Venturino, X. Wang, and M. Lops, "Multiuser detection for cooperative networks and performance analysis," IEEE Trans. Signal Process., vol. 54, no. 9, pp. 3315-3329, Sept. 2006.

[7] A. K. Sadek, W. Su, and K. J. R. Liu, "Multinode cooperative communications in wireless networks," IEEE Trans. Signal Process., vol. 55, no. 1, pp. 341-355, Jan. 2007.

[8] K. G. Seddik, A. K. Sadek, W. Su, and K. J. R. Liu, "Outage analysis and optimal power allocation for multi-node relay networks," IEEE Signal Processing Letters, vol. 14, no. 6, pp. 377-380, Jun. 2007.

[9] T. Wang and G. B. Giannakis, "Complex field network coding for multiuser cooperative communications," IEEE J. Sel. Areas Commun., vol. 26, no. 2, pp. 561-571, Apr. 2008.

[10] "IEEE Standard for Local and Metropolitan Area Networks: Wireless LAN Medium Access Control (MAC) and Physical Lyer (PHY) Specification," IEEE Std 802.11-2007.

[11] M. K. Simon and M. S. Alouini, "A unified approach to the performance analysis of digital communication over generalized fading channels," Proc. IEEE, vol. 86, no. 9, pp. 1860-1877, Sep. 1998.

[12] A. Leon-Garcia, Probability and Random Processes for Electrical Engineering. USA: Addison Wesley Longman, 2nd ed., 1994. 